\title{
Construção e Validação de podcast como tecnologia educacional para prevenção
}

\section{da Hemorragia Pós-parto}

\author{
Construction and Validation of podcast as educational technology for preventing Postpartum
}

Hemorrhage

Construcción y Validación de podcast como tecnologia educativa para prevenir la Hemorragia

Posparto

Recebido: 12/02/2021 | Revisado: 21/02/2021 | Aceito: 24/02/2021 | Publicado: 03/03/2021

\author{
Alessandra da Silva Mota \\ ORCID: https://orcid.org/0000-0001-9458-2264 \\ Universidade do Estado do Pará, Brasil \\ E-mail: ale.thur@outlook.com \\ Jessyca Santos Cavalcante dos Reis \\ ORCID: https://orcid.org/0000-0003-0280-1357 \\ Universidade do Estado do Pará, Brasil \\ E-mail: jessyca_amada_santos@hotmail.com \\ Sarah Lais Rocha \\ ORCID: https://orcid.org/0000-0002-3753-9507 \\ Universidade do Estado do Pará, Brasil \\ Faculdade Carajás, Brasil \\ E-mail: sarahlaisrocha@gmail.com \\ Gisele Rodrigues de Carvalho Oliveira \\ ORCID: https://orcid.org/0000-0002-1229-0215 \\ Universidade do Estado do Pará, Brasil \\ E-mail: gisele.r.carvalho@bol.com.br \\ Robson José de Souza Domingues \\ ORCID: https://orcid.org/0000-0001-5419-2878 \\ Universidade do Estado do Pará, Brasil \\ E-mail: domingues@uepa.br
}

\begin{abstract}
Resumo
Objetivo: Construir e validar uma tecnologia educacional do tipo podcast relacionado à prevenção da mortalidade materna por hemorragia pós-parto para alunos do curso de medicina. Metodologia: Após a aprovação do protocolo de estudo pelo Comitê de Ética em Pesquisa se iniciou um estudo de validação de tecnologia do tipo pesquisa de desenvolvimento metodológico com abordagem quantitativa. Para a construção do podcast educacional foi realizado um levantamento bibliográfico, em seguida ocorreu a definição dos roteiros técnicos e a construção de cinco episódios da série podcast. Então, foram selecionados os 32 juízes-especialistas, para apreciação e validação desta tecnologia. Considerou-se os intervalos de validação de acordo com o número de juízes e a escala likert de 5 pontos, entre 2.241 a 3.360 pontos: validado e o intervalo de classificação para o Alfa de Cronbach > 0,80 como excelente consistência interna. Resultados: A validação do produto foi executada por meio de questionário com três domínios (objetivos, estrutura/apresentação e relevância), alcançando um valor Alfa de Cronbach de 0.873, classificado como excelente consistência. Na avaliação geral o podcast atingiu 3.228 pontos, de um valor possível de 3.360 pontos (96.1\%), sendo validado pelos avaliadores. Conclusão: O estudo evidenciou que o podcast foi validado por juízes-especialistas e considerado adequado para ser utilizado pelos estudantes de medicina, pressupondo sua utilização como instrumento tecnológico educacional de saúde sobre a prevenção da mortalidade materna por Hemorragia Pós-Parto (HPP).
\end{abstract}

Palavras-chave: Estudos de validação; Educação em saúde; Hemorragia pós-parto; Hora de ouro; Mortalidade materna.

\begin{abstract}
Objective: To build and validate a podcast-type educational technology related to the prevention of maternal mortality from postpartum hemorrhage for medical students. Methodology: After the approval of the study protocol by the Research Ethics Committee, a study to validate technology of the type of methodological development research with a quantitative approach was initiated. For the construction of the educational podcast, a bibliographic survey was carried out, followed by the definition of technical scripts and the construction of five episodes of the podcast series. Then, the 32 expert judges were selected, followed by the validation of this technology. The validation intervals were considered according to the number of judges and the 5-point likert scale, from 2,241 to 3,360 points: validated and the classification interval for Cronbach's alpha> 0.80 as excellent internal consistency. Results: The product validation was performed
\end{abstract}


through a questionnaire with 3 domains (objectives, structure / presentation and relevance), reaching a Cronbach's Alpha value of 0.873 , classified as excellent consistency. In the overall evaluation, the podcast reached 3,228 points, out of a possible value of 3,360 points $(96.1 \%)$, being validated by the evaluators. Conclusion: The study showed that the podcast was validated by expert judges and considered suitable for use by medical students, assuming its use as a technological health tool on the prevention of maternal mortality from Postpartum Hemorrhage (PPH).

Keywords: Validation studies; Health education; Postpartum hemorrhage; Golden hour; Maternal mortality.

\section{Resumen}

Objetivo: Construir y validar una tecnología educativo tipo podcast relacionada con la prevención de la mortalidad materna por hemorragia posparto para estudiantes de medicina. Metodología: Luego de la aprobación del protocolo de estudio por parte del Comité de Ética en Investigación, se inició un estudio para validar tecnología del tipo de investigación de desarrollo metodológico con enfoque cuantitativo. Para la construcción del podcast educativo se realizó un relevamiento bibliográfico, seguido de la definición de guiones técnicos y la construcción de 5 episodios de la serie de podcasts. Luego, se seleccionaron los 32 jueces expertos, seguido de la validación de esta tecnología. Los intervalos de validación se consideraron según el número de jueces y la escala Likert de 5 puntos, de 2.241 a 3.360 puntos: validado y el intervalo de clasificación para alfa de Cronbach> 0,80 como excelente consistencia interna. Resultados: La validación del producto se realizó mediante un cuestionario con 3 dominios (objetivos, estructura / presentación y relevancia), alcanzando un valor de Alfa de Cronbach de 0.873, clasificado como excelente consistencia. En la evaluación global, el podcast alcanzó los 3.228 puntos, de un valor posible de 3.360 puntos (96,1\%), siendo validado por los evaluadores. Conclusión: El estudio mostró que el podcast fue validado por jueces expertos y considerado apto para ser utilizado por estudiantes de medicina, asumiendo su uso como una herramienta tecnológica de salud en la prevención de la mortalidad materna por hemorragia posparto (HPP).

Palabras clave: Estudios de validación; Educación para la salud; Hemorragia post parto; Hora dorada; Mortalidad maternal.

\section{Introdução}

Morte materna compreende os óbitos ocorridos durante a gestação ou até 42 dias após o término desta. Dados obtidos através do painel de monitoramento da mortalidade materna, demonstram que no ano de 2019 a quantidade de óbitos maternos, com indicador materno declarado e local de registro óbitos por ocorrência, na faixa etária de 10 a 49 anos no Brasil foi de 848 , na região norte 119, no Pará 50, na região Carajás 7 e 4 casos em Marabá. No que tange ao número de mortes maternas por hemorragia, no Brasil houve 108, no Norte 22, no Pará 9, na região Carajás 3 e em Marabá 2 casos (Brasil, 2019).

A concepção do podcast ocorreu em 2004, quando o ex-Vídeo Jockey da MTV Adam Curry e o programador Dave Winer criaram um software que permitia descarregar automaticamente transmissões de rádio na internet diretamente para os seus iPods, tornando-se uma forma eficiente para publicação de arquivos digitais (áudio, vídeo, imagem...), através de atualizações para o computador (Mendonça \& Duarte, 2010).

Podcast "é um meio veloz de distribuir sons pela internet, um neologismo que funde duas palavras: iPod, o tocador de arquivos digitais de áudio da Apple, e broadcast, que significa transmissão em inglês. O podcast pode ter vários programas, ou episódios, como se fosse um seriado. Os arquivos ficam hospedados em um endereço na internet e, por download, chegam ao computador pessoal ou tocador" (Foschini \& Taddei, 2006).

Ademais, a divulgação, por meio de algum site ou blog, possui um texto de lançamento e ocorre de forma democrática, gratuita e rápida, assegurando essa incrível liberdade aos ouvintes. Uma vez que são produzidos sob demanda e não são transmitidos em tempo real, permitindo assim que sejam ouvidos posteriormente no momento mais adequado para o assinante (Luiz \& Assis, 2010).

“Em princípio, podcasts também são programas de vídeo distribuídos dessa forma. Porém, no Brasil, acabou ficando uma certa "separação" informal que classifica os programas de áudio como podcast e os de vídeo como videocast”" (Luiz,2014).

Outrossim, durante a produção do arquivo, é comum que haja interação entre os participantes do episódio e/ou com o público, gerando debates, discussões sobre o tema previamente escolhido, esta é uma das principais características do podcast. 
Atualmente, há uma variedade de temas apresentados, cultura, arte, cinema, literatura, música, fotografia, videogames, história, educação, ciência, saúde, notícias, idiomas, marketing e entre outros (Silva, 2019).

Além disso, a elevada quantidade de podcast disponíveis hoje é justificada por alguns pontos relacionados à sua produção, pois é bem acessível financeiramente, não é necessário um alto investimento tecnológico para isso, assim como não é preciso ter muito conhecimento técnico. Os arquivos necessitam ser pequenos em volume de dados, para isso são utilizados programas de compressão, os quais não modificam muito a qualidade do áudio do produto, com o objetivo de que todos possam realizar o download, sendo o MP3 o formato geralmente escolhido. A disponibilidade gratuita de vários softwares na internet também são fatores que contribuem para a grande disseminação do podcast (Vanassi, 2007).

Neste Contexto, a Organização Pan-Americana de Saúde (OPAS) em associação com a Organização Mundial da Saúde (OMS) e Ministério da Saúde (MS), lançaram a estratégia Zero Morte Materna por Hemorragia (0MMxH), na qual objetiva-se a prevenção e conduta da Hemorragia Pós-Parto (HPP), organizando curso com simulação para capacitar os profissionais da saúde no enfrentamento dessa situação, o primeiro treinamento no Brasil ocorreu em 2016.

Assim, este estudo objetivou construir e validar uma tecnologia educacional do tipo podcast relacionado à prevenção da mortalidade materna por hemorragia pós-parto para alunos do curso de medicina. Cujos objetivos específicos foram: construir podcasts com embasamento teórico para a prevenção da mortalidade materna por hemorragia pós-parto, verificar se os podcasts são um instrumento estatisticamente válido para ser usado pelo público-alvo segundo os juízes especialistas, validar o conteúdo do podcast educacional em saúde por médicos/enfermeiros especialistas em obstetrícia/saúde da mulher e por profissionais da área de mídias digitais, podcast e/ou tecnologia educacional e divulgar os podcasts entre os estudantes de medicina da Universidade do Estado do Pará- UEPA campus VIII - Marabá.

\section{Metodologia}

Após a aprovação do protocolo de estudo pelo comitê de ética em pesquisa, da Universidade do Estado do Pará campus VIII-Marabá, com número CAEE 36010920.1.0000.8607 se iniciou um estudo de validação de tecnologia do tipo pesquisa de desenvolvimento metodológico com abordagem quantitativa.

Um estudo de desenvolvimento metodológico envolve investigações dos métodos de obtenção e organização de dados e condução de pesquisas rigorosas, tratam do desenvolvimento, da validação e da avaliação de ferramentas e métodos de pesquisa. As crescentes demandas por avaliações de resultados sólidas e confiáveis, testes rigorosos de intervenção e procedimentos sofisticados de obtenção de enfermeiros pesquisadores (Polit \& Beck, 2011).

Para validação dessa tecnologia de educação em saúde foi necessário que juízes avaliassem as características do podcast, por meio de escores estabelecidos, desde o conteúdo até a qualidade da mídia utilizada. E, dessa maneira, houve quantificação da concordância deles acerca da validação do conteúdo, isto é, abordagem quantitativa, a qual permite analisar o grau de precisão do instrumento (Nascimento et al., 2012).

Por meio da Escala Likert (questões fechadas) o método qualitativo se transforma em quantitativo, na qual são feitas afiramções e os juízes-especialistas escolhem um número de 1 a 5 relacionado com graus de concordância com a mesma. O número 1 corresponderia à discordância completa, enquanto que o número 5 à concordância completa e os outros números são situações intermediárias. Observa-se também que as respostas são agrupadas e ao possuir dados numéricos pode ser realizada uma análise estatística (Pereira, Shitsuka, Parreira \& Shitsuka, 2018). 
Para a validação, com nível de confiança do estudo de $99 \%$ foram selecionados 32 juízes da área de saúde, educação, comunicação e tecnologia na educação a concordância entre eles de $95 \%$ e erro amostral considerável aceitável de $10 \%$, segundo dados estatísticos realizados previamente (Lopes et al., 2013).

Critérios de inclusão: cada juiz-especialista, tanto da área da saúde quanto da área de tecnologia e educação atingiu no mínimo 5 pontos (Adaptação de Fehering 1994).

Ter graduação em medicina/enfermagem (critério obrigatório) ou ter graduação em comunicação em mídias digitais ou área da educação (2 pontos), ser mestre/doutor (1 ponto), os critérios a seguir são referentes a área da obstetrícia/saúde da mulher, ter residência/especialização ( 2 pontos), ser mestre/doutor com dissertação ( 2 pontos), os demais itens também tem relação com hemorragia pós-parto, ter pesquisas/artigos ( 2 pontos), ter prática clínica/docente de pelo menos 1 ano (1 ponto), ter participação em cursos, congressos ou capacitações (1 ponto), ter orientado trabalhos (1 ponto), participar de banca examinadora de trabalhos (1 ponto), aceitar sua participação na pesquisa (1 ponto) e assinar o termo de consentimento de livre esclarecido (tcle).

Critério de exclusão o juiz que não respondeu a alguma das etapas de validação, estava de férias ou afastado do serviço durante o período da pesquisa.

\subsection{Coleta de dados}

Foi realizada uma revisão integrativa de literatura nas bases de dados (PUBMED, SCIELO, LILACS, ERIC) para a base teórica deste estudo. Na sequência se iniciou a construção do produto, na fase de pré-produção, o grupo definiu os roteiros técnicos, com os assuntos mais pertinentes sobre prevenção de mortalidade materna por hemorragia pós-parto para serem abordados, divisão didática mais adequada para melhor compreensão do público, quem iria direcionar o roteiro durante a gravação e a seleção dos convidados, os quais foram revisados por uma ginecologista e obstetra.

A fase da produção foi realizada por meio do aplicativo "anchor“ na versão 3.43 .0 para android, desde a gravação das falas, a edição dos episódios, até a publicação da série de cinco podcasts na plataforma spotify.

Na etapa seguinte foi realizada a seleção dos juízes-especialistas a partir dos currículos lattes disponíveis na plataforma lattes do portal do cnpq, de acordo com os critérios de inclusão. Pesquisou-se por palavras-chave como obstetrícia, hemorragia pós-parto, tecnologia educacional e/ou podcast, na opção busca simples, houve compartilhamento do link de acesso ao google forms o qual continha o tcle, perguntas relacionadas à caracterização destes juízes, link para redirecionar aos podcasts e o instrumento de validação.

Ademais, para escolha da amostragem foi utilizada a técnica "bola de neve", na qual cada juiz selecionado indica pelo menos outro que atinja a pontuação mínima dos critérios de inclusão. As avaliações retornaram aos pesquisadores por volta de 14 dias.

\subsection{Análise de dados}

As informações da caracterização amostral foram apuradas e digitadas em planilha elaborada no software microsoft ${ }^{\circledR}$ office excel $^{\circledR}$ 2016. Na aplicação da estatística descritiva, foram construídas tabelas e gráficos para apresentação dos resultados e calculadas as medidas de posição como média aritmética e desvio padrão.

A avaliação de validação foi realizada por meio da Escala de Likert de 5 pontos, sendo os intervalos de validação de acordo com o número de juízes, o seguinte: de 0 a 1.120 pontos: não validado, de 1.121 a 2.240 pontos: validado com ressalvas e de 2.241 a 3.360 pontos: validado.

A consistência do questionário foi avaliada por meio do Alfa de Cronbach, calculado através da fórmula: 


$$
\alpha=\frac{k}{k-1}\left(1-\frac{\sum_{i=1}^{k} s_{i}^{2}}{s_{\text {iens }}^{2}}\right)
$$

O intervalo de classificação para o alfa de cronbach com a consistência interna foi: > 0,80 (excelente); 0,61 a 0,80 (substancial); 0,41 a 0,60 (moderado); 0,21 a 0,40 (razoável) e < 0,21 (inaceitável).

\subsection{Riscos e benefícios}

O estudo foi desenvolvido em condições de rotina, os riscos envolvendo os participantes foram relacionados com a exposição indevida de informações pessoais, vazamento de informações e quebra do anonimato e possível identificação dos pesquisados. Como precaução todas as informações foram codificadas, sendo utilizadas somente pelo pessoal autorizado, mantendo-se o sigilo das informações coletadas e anonimato dos envolvidos, de forma a preservar a integridade dos participantes da pesquisa.

A pesquisa ajudará a divulgar informações essenciais quanto à prevenção da mortalidade materna por hemorragia pósparto no município de marabá e região. Dessa forma, contribuirá para o desenvolvimento de ações intervencionistas que visem diminuir o número de casos de morte materna por hemorragia pós-parto. A divulgação dos podcasts à comunidade acadêmica possibilitará atualização e capacitação sobre este assunto, a fim de reduzir a prevalência deste agravo.

\subsection{Aspectos éticos e legais}

O estudo obedeceu à resolução $\mathrm{n}^{\circ}$ 466/2012 do conselho nacional de saúde (CNS) que regulamenta a pesquisa envolvendo seres humanos. O estudo foi conduzido após a submissão e aprovação pelo CEP/Campus VIII e todos os participantes assinaram o termo de consentimento livre e esclarecido (TCLE).

\section{Resultados}

Entre os juízes avaliadores do produto, a maioria foi do sexo feminino (84.4\%). As idades variaram entre 25 e 59 anos, com média aritmética de 38.2 anos. O tempo de formação dos profissionais variou de 01 a 35 anos e média aritmética de 13.2 anos, como mostra a Tabela 1. 
Tabela 1: Perfil dos juízes avaliadores do Podcast, Marabá, 2021.

\begin{tabular}{|c|c|c|}
\hline Perfil dos avaliadores & Frequência & $\%(n=32)$ \\
\hline \multicolumn{3}{|l|}{ Sexo } \\
\hline Feminino & 27 & $84.4 \%$ \\
\hline Masculino & 5 & $15.6 \%$ \\
\hline \multicolumn{3}{|l|}{ Faixa etária (anos) } \\
\hline$<30$ & 5 & $15.6 \%$ \\
\hline 30 a 39 & 14 & $43.8 \%$ \\
\hline 40 a 49 & 9 & $28.1 \%$ \\
\hline$>=50$ & 4 & $12.5 \%$ \\
\hline Mínima / Média / Máxima & $25 / 38.2 / 59$ & \\
\hline \multicolumn{3}{|l|}{ Tempo de formação } \\
\hline$<10$ & 14 & $43.8 \%$ \\
\hline 10 a 19 & 8 & $25.0 \%$ \\
\hline 20 a 29 & 8 & $25.0 \%$ \\
\hline$>=30$ & 2 & $6.3 \%$ \\
\hline Mínimo / Média / Máximo & $01 / 13.2 / 35$ & \\
\hline
\end{tabular}

Fonte: Protocolo eletrônico aplicado.

Entre os juízes avaliadores, houve proporção estatisticamente significante $(* \mathrm{p}<0.0001)$ de profissionais com especialização concluída (65.6\%). A área de atuação mais frequente foi a da Medicina em Ginecologia e Obstetrícia (59.4\%) e as funções de Médico (a) obstetra (37.5\%) e Docentes (34.4\%), conforme tabela 2. 
Tabela 2: Formação e atuação profissional dos juízes avaliadores, Marabá, 2021.

\begin{tabular}{lll}
\hline Formação e atuação profissional & Frequência & \% (n = 32) \\
\hline Titulação & 21 & $65.6 \%$ \\
Especialização concluída* & 5 & $15.6 \%$ \\
Mestrado em andamento & 3 & $9.4 \%$ \\
Mestrado concluído & 3 & $9.4 \%$ \\
Doutorado concluído & & \\
Área de formação & 19 & $59.4 \%$ \\
Medicina/ Ginecologia e Obstetrícia* & 8 & $25.0 \%$ \\
Enfermagem/ Obstetrícia & 2 & $6.3 \%$ \\
Enfermagem & 1 & $3.1 \%$ \\
Jornalismo & 1 & $3.1 \%$ \\
Letras & 1 & $3.1 \%$ \\
Saúde coletiva & & $3.1 \%$ \\
Função na Instituição & 12 & $34.4 \%$ \\
Médico(a) Obstetra & 11 & $25.0 \%$ \\
Docente & 8 & $3.1 \%$ \\
Enfermeiro(a) Obstetra & 1 & \\
Comunicadora & & \\
\hline
\end{tabular}

*p $<0.0001$ Teste G Aderência - Fonte: Protocolo eletrônico aplicado.

Entre os juízes-especialistas da área de saúde, a maioria significante participa de cursos, congressos ou capacitações referentes à obstetrícia/saúde da mulher e/ou hemorragia pós-parto (100\%), possuem residência/especialização na área de ginecologia e obstetrícia/saúde da mulher (93.3\%) e possuem prática clínica/docente de pelo menos 1 ano em obstetrícia/saúde da mulher (90.0\%).

Pesquisas/artigos publicadas na área de obstetrícia/saúde da mulher e/ou hemorragia pós-parto e orientação de trabalhos na área de saúde da obstetrícia/saúde da mulher e/ou hemorragia pós-parto, alcançaram a mesma proporção entre os juízes/avaliadores (43.3\%), como mostra a Tabela 3. 
Tabela 3: Informações sobre a residência médica, Marabá, 2021.

\begin{tabular}{lll}
\hline Juízes-especialistas da área da saúde & Frequência & \% (n= 30) \\
\hline Graduação em medicina ou enfermagem* & 30 & $100.0 \%$ \\
$\begin{array}{l}\text { Cursos, congressos ou capacitações referentes à obstetrícia/saúde da } \\
\text { mulher e/ou hemorragia pós-parto* }\end{array}$ & 30 & $100.0 \%$ \\
$\begin{array}{l}\text { Residência/especialização na área de ginecologia e obstetrícia/saúde da } \\
\text { mulher* }\end{array}$ & 28 & $93.3 \%$ \\
$\begin{array}{l}\text { Tem prática clínica/docente de pelo menos } 1 \text { ano em obstetrícia/saúde } \\
\text { da mulher* }\end{array}$ & 27 & $90.0 \%$ \\
$\begin{array}{l}\text { Tem pesquisas/artigos publicadas na área de obstetrícia/saúde da } \\
\text { mulher e/ou hemorragia pós-parto }\end{array}$ & 13 & $43.3 \%$ \\
$\begin{array}{l}\text { Orientou trabalhos na área de saúde da obstetrícia/saúde da mulher e/ou } \\
\text { hemorragia pós-parto }\end{array}$ & 13 & $43.3 \%$ \\
$\begin{array}{l}\text { Participou de banca examinadora de trabalhos sobre obstetrícia/saúde } \\
\text { da mulher e/ou hemorragia pós-parto }\end{array}$ & 9 & $6.7 \%$ \\
$\begin{array}{l}\text { Possui mestrado ou doutorado } \\
\text { Mestre/doutor com dissertação na área de obstetrícia/saúde da mulher }\end{array}$ & 2 & $30.0 \%$ \\
\hline
\end{tabular}

*p $<0.0001$ Teste G Aderência - Fonte: Protocolo eletrônico aplicado.

A validação do produto foi realizada através de questionário com três domínios que avaliaram os Objetivos, a Estrutura/Apresentação e a Relevância do podcast. O questionário alcançou um valor Alfa de Cronbach de 0.873 , classificado como Excelente Consistência.

Todas as afirmações sobre os objetivos, alcançaram percentual de aprovação acima de 95.0\%. Todas as afirmações que o produto pode ser utilizado como instrumento de educação em saúde no processo ensino-aprendizagem e que responde às dúvidas sobre prevenção de mortalidade materna por HPP, alcançaram as maiores proporções (99.4\% e $98.1 \%$ respectivamente). No geral, os objetivos alcançaram 932 pontos, de um total possível de 960 pontos, sendo uma aprovação de $97.1 \%$, conforme Gráfico 1. 
Research, Society and Development, v. 10, n. 3, e3610312913, 2021

(CC BY 4.0) | ISSN 2525-3409 | DOI: http://dx.doi.org/10.33448/rsd-v10i3.12913

Gráfico 1: Pontuação alcançada na avaliação dos Objetivos do Podcast, Marabá, 2021.

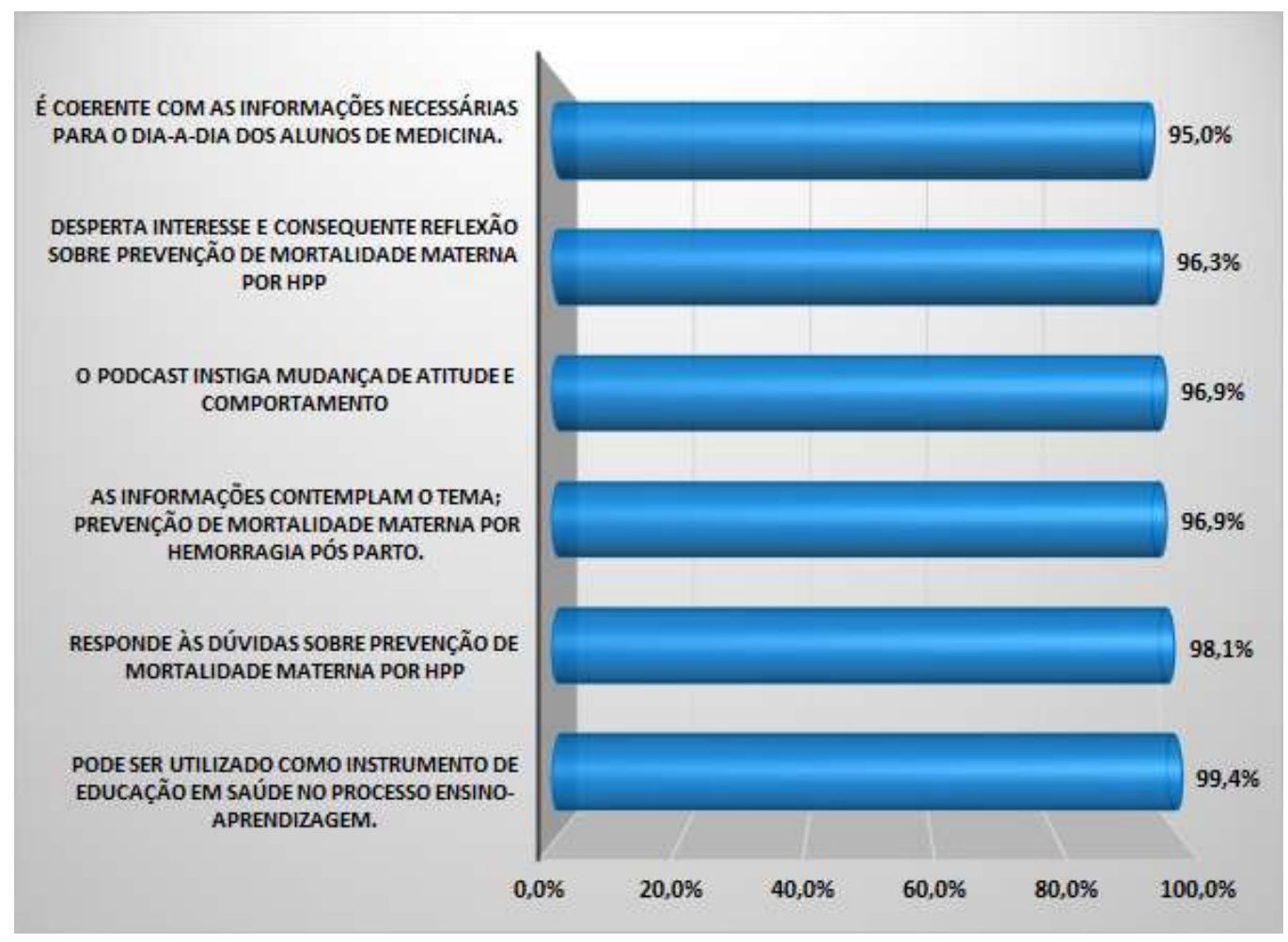

Fonte: Protocolo eletrônico aplicado.

$\mathrm{Na}$ avaliação dos juízes/avaliadores a respeito da Estrutura e Apresentação do podcast, a afirmação que o tema é relevante na atualidade, alcançou $100 \%$ de concordância. As maiores proporções de aprovação, depois da citada acima, ficaram as afirmações de que o título do podcast é compatível com o assunto abordado (97.5\%) e que possui conteúdo adequado para estudantes de medicina (98.1\%).

A menor proporção de aprovação ficou com a questão que afirmava que o produto promove interação com o públicoalvo (88.1\%). No geral, a Estrutura e Apresentação alcançou 1677 pontos, de um total possível de 1760 pontos, sendo uma aprovação de $95.3 \%$, conforme Gráfico 2 . 
Gráfico 2: Pontuação alcançada na avaliação da Estrutura e Apresentação do Podcast, Marabá, 2021.

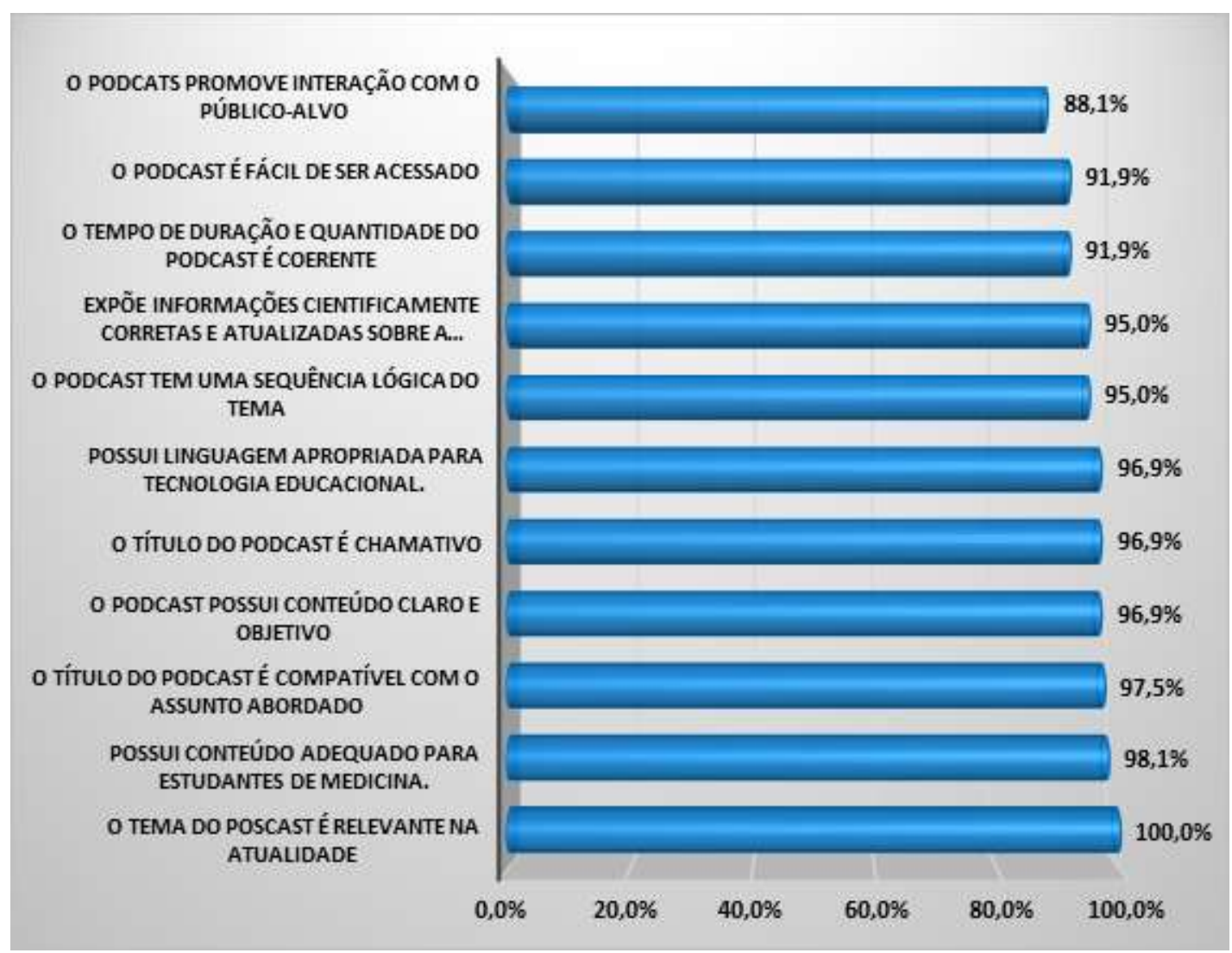

Fonte: Protocolo eletrônico aplicado.

A avaliação da Relevância do podcast foi de 619 pontos, de um total possível de 640 pontos, sendo uma aprovação de 96.7\%. A questão que afirmava que o produto aborda informações que os alunos de medicina devem estar cientes, alcançou a maior proporção de concordância entre as demais afirmações deste domínio (99.4\%).

As afirmações que o Podcast divulga aspectos-chaves, relacionados à prevenção de mortalidade materna por HPP e está adequado para serem utilizados pelos alunos de medicina, alcançaram a mesma proporção de aprovação (97.5\% ambas). A menor proporção de concordância ficou com a afirmação de que o produto permite a sua utilização em diferentes contextos e realidades (92.5\%), conforme Gráfico 3. 
Research, Society and Development, v. 10, n. 3, e3610312913, 2021

(CC BY 4.0) | ISSN 2525-3409 | DOI: http://dx.doi.org/10.33448/rsd-v10i3.12913

Gráfico 3: Pontuação alcançada na avaliação da Relevância do Podcast, Marabá, 2021.

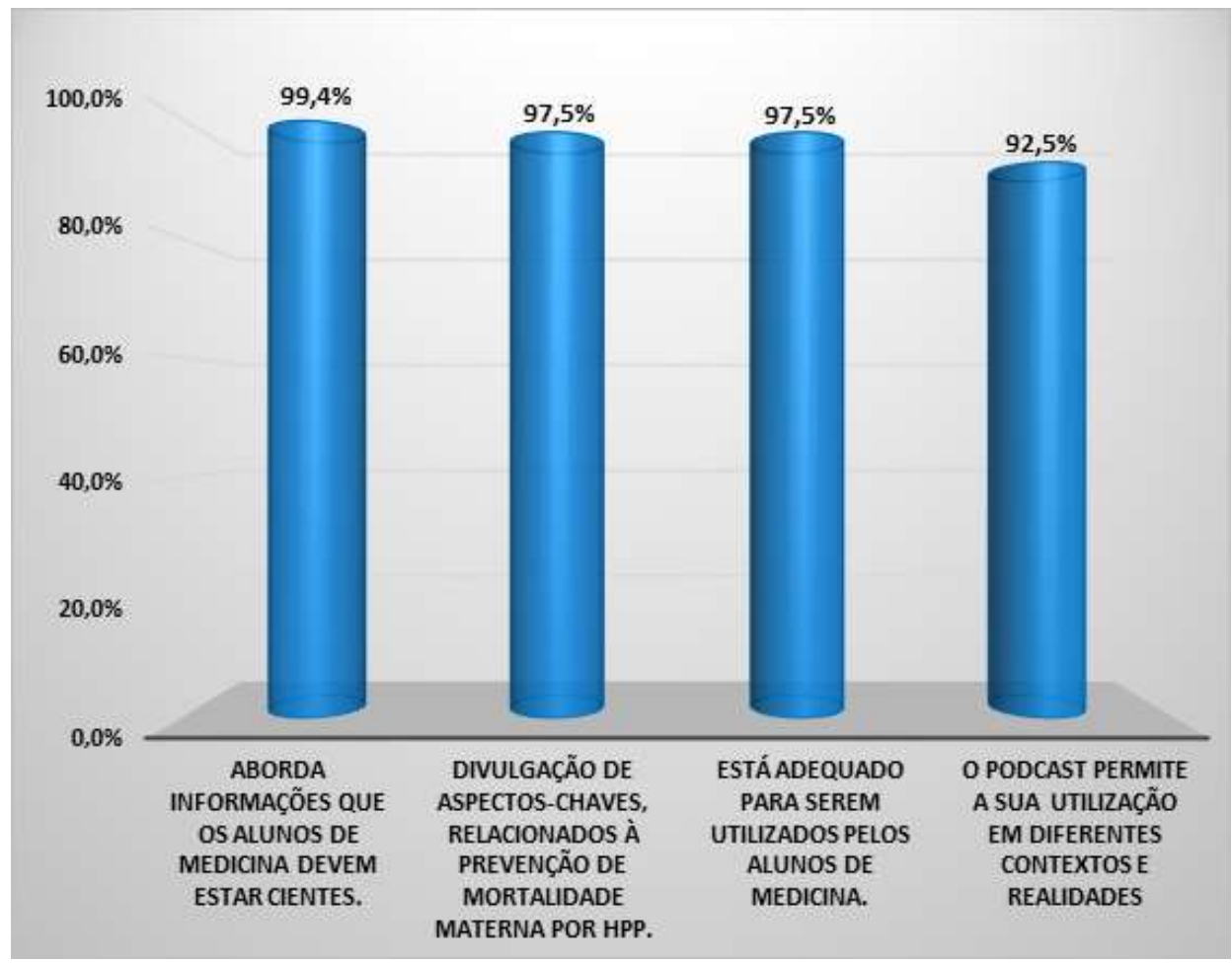

Fonte: Protocolo eletrônico aplicado.

$\mathrm{Na}$ avaliação geral, considerando os domínios analisados, o podcast alcançou 3.228 pontos, de um valor possível de 3.360 pontos (96.1\%), sendo considerado validado pelos juízes/avaliadores, como demonstrado no Gráfico 4.

Gráfico 4: Avaliação geral do Podcast, Marabá, 2021.

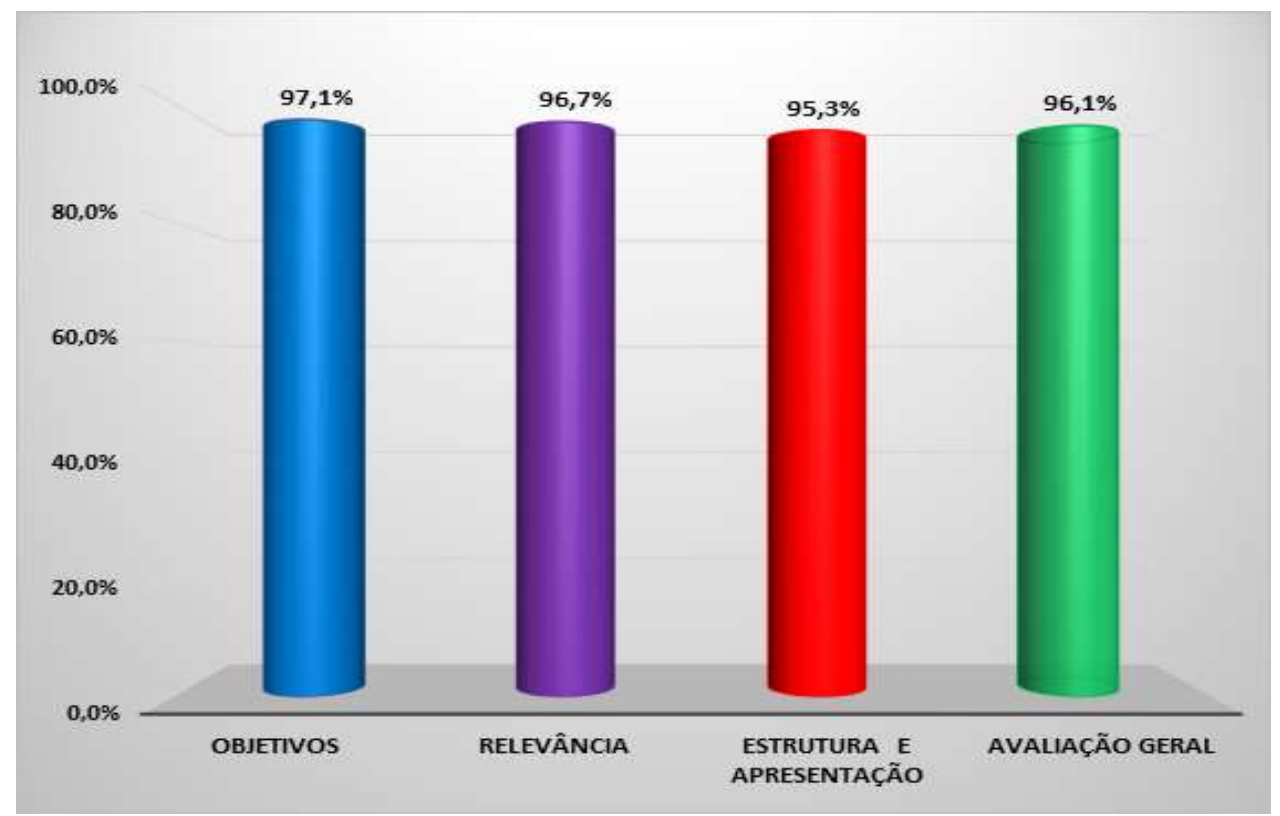

Fonte: Protocolo eletrônico aplicado. 


\section{Discussão}

Com o avanço das tecnologias digitais, o contexto educacional de estudantes de medicina e profissionais da área da saúde, segue para uma direção mais moderna. Assim, tornam-se necessárias alternativas que favoreçam esse aprendizado, por exemplo o uso de podcast como tecnologia educacional. A tecnologia podcast se destaca cada vez mais, devido às suas características particulares, como flexibilidade, diversidade de assuntos, interatividade e possiblidade de usar estes recursos em diferentes locais (Vasconcelos et al., 2020).

Ademais, o uso de podcast também está sendo difundido no meio educacional com maior frequência, inclusive sobre seu processo de construção. Por exemplo, o projeto Metacast teve como objetivo central a elaboração de materiais didáticos que auxiliassem o educador no processo de familiarização com o podcast e suas etapas de criação (Veloso et al., 2019). Assim como o BHcast que apresenta o caminho percorrido na produção de um podcast com o objetivo de disseminar a informação científica no ambiente universitário (Santos, 2020).

Outra experiência inovadora foi descrita no projeto LaSalleCast, que tem como objetivo central o de organizar a produção de podcasts de cunho educacional, abordando conteúdos de disciplinas dos cursos à distância (EAD) de uma universidade (Saraiva et al., 2018). Dessa forma, revela-se a importância da construção e validação de tecnologias educacionais, dentro das mais diversas áreas, a exemplo da educação em saúde, as quais auxiliam no processo de ensino-aprendizagem, principalmente no contexto das metodologias ativas do conhecimento.

A hemorragia pós-parto é uma das principais causas evitáveis de morte materna. Contudo, o nosso estado (Pará) ainda apresenta números alarmantes de óbitos maternos por hemorragia pós-parto. Então, utilizou-se uma tecnologia educacional (podcast), afim de divulgar informações coerentes acerca da prevenção da mortalidade materna por hemorragia pós-parto, entre os estudantes de medicina e profissionais da área da saúde, com intuito de diminuir os casos de óbito materno relacionados a isso.

Esta tecnologia educacional em saúde, intitulada “ MedCast - Hora de ouro" - possui uma série de cinco episódios, abordando no episódio 1: A epidemiologia, definição e classificação de morte materna por hemorragia pós-parto; Episódio 2: Fatores de risco associados e os 4T’s da HPP; Episódio 3: O papel da equipe e a estimativa da perda de sanguínea; Episódio 4: Prevenção da mortalidade materna por hemorragia pós-parto; Episódio 5: Hora de ouro.

O podcast como ferramenta de apoio à redução de óbito materno por hemorragia pós-parto, reforça o tripé da prevenção, diagnóstico e tratamento precoce. Uma vez que é preconizado o manejo ativo do terceiro período do trabalho de parto, com a ocitocina sendo a principal terapia deste processo. Associado ao papel de monitorização e acompanhamento da puérpera pela equipe multidisciplinar, com o objetivo de detectar qualquer anormalidade e sinal de alerta nesse período pós-parto. E por fim, o tratamento mais precocemente possível com medidas medicamentosas, não medicamentosas e cirúrgicas, para evitar um desfecho desfavorável que possa levar ao óbito materno.

Diante disso, é esperado que informações divulgadas pelos podcasts sejam assimiladas por todos que, de alguma forma, prestam assistência ao trabalho de parto, visto que estas medidas preventivas são imprescindíveis para a redução da prevalência de hemorragia pós-parto. Como a ocitocina após o parto que reduz mais de $50 \%$ dos casos de hemorragia por atonia uterina (10 UI IM). Além disso é importante que seja feito o clampeamento oportuno, tração controlada do cordão umbilical e vigilância/massagem uterina após dequitação.

No âmbito da saúde vários podcasts vem surgindo, como o Café com saúde, são aulas desenvolvidas no formato de áudio direcionadas a estudantes de nível superior dos cursos da área da saúde (medicina, enfermagem, biologia e odontologia), abordando temas como histologia, imunologia, genética, biologia celular e as patologias mais prevalente no SUS (Gomes et al., 
2019). Assim como o projeto Contêiner em saúde, o qual divulga informações de promoção, prevenção e educação em saúde para as Populações Vulneráveis (Santos, 2016).

Um importante estudo que construiu e validou o podcast como tecnologia educacional na área da saúde foi Muniz (2017), no qual estudantes de enfermagem promoveram educação em saúde sobre hanseníase. A média do I-CVI (Item-level Content Validity Index) para este podcast educacional foi de 0,89. Já o S-CVI (Scale-level Contente Validity Index) que corresponde à média aritmética da proporção dos itens que receberem avaliação de concordo ou concordo totalmente do total de juízes foi igual a 0,90 .

Em desconformidade com Muniz (2017), no qual verifica-se alguns itens com menos de $80 \%$ e vários com 100\% de concordância, neste presente trabalho todos os critérios obtiveram escore acima de $85 \%$ embora o único que atingiu concordância por completo foi a afirmação que o tema é relevante na atualidade referente à estrutura e apresentação.

Igualmente ao estudo de Muniz (2017), no qual apresentou escore quanto ao conteúdo de 95\%, quanto à aparência de $82 \%$ e quanto à funcionalidade de $86 \%$ e quanto ao ambiente sonoro de $95 \%$, individualmente cada uma das dimensões também foi validado com escores acima de $80 \%$, apresentando os seguintes valores: objetivos (97,1\%), estrutura e apresentação (96,7\%) e relevância $(95,3 \%)$.

Semelhante a Muniz (2017), no qual as menores pontuações foram relacionadas à aparência, sendo os itens do tempo final do podcast e a duração de alguns diálogos, nestes resultados obtidos observa-se como segundo menor escore o item de que tempo de duração e quantidade do podcast é coerente $(91,9 \%)$ relacionado à estrutura e apresentação. Entretanto, a menor proporção de aprovação ficou com a questão de estrutura e apresentação que afirmava que o produto promove interação com o público-alvo (88.1\%).

No capítulo anterior (resultados) todas as afirmações sobre os objetivos alcançaram percentual de aprovação acima de 95.0\%. Mostra-se que as maiores proporções de concordância no que tange aos objetivos foram de que o produto pode ser utilizado como instrumento de educação em saúde no processo ensino-aprendizagem e que responde às dúvidas sobre prevenção de mortalidade materna por HPP, alcançando as maiores proporções (99.4\% e $98.1 \%$ respectivamente). Essa consideração é fundamental para o estudo, uma vez que estudantes de medicina e profissionais de saúde podem reforçar conhecimentos adquiridos através do podcast para atuarem diante desta emergência obstétrica.

De mesmo modo que em Muniz (2017), no qual um dos critérios que obtiveram escores bem elevados foi o conteúdo (95\%) e afirma que o conteúdo atende uma possível situação de educação em saúde (100\%), o conteúdo é coerente com uma prática educacional em saúde (95\%), o conteúdo é relevante para a educação em saúde (100\%) e o podcast é esclarecedor sobre hanseníase (100\%).

Em suma, dos aspectos avaliados pelos juízes, quanto aos objetivos a serem atingidos com a utilização do podcast, destacaram que as informações contidas no material educacional são coerentes e válidas para serem usadas pelo público-alvo.

\section{Conclusão}

O estudo evidenciou que o podcast "MedCast-Hora de ouro" foi validado em conteúdo e relevância por juízes especialistas, classificado como excelente consistência (valor Alfa de Cronbach de 0.873). Ademais, foi considerado adequado para ser utilizado pelos estudantes de medicina, pressupondo sua utilização como instrumento tecnológico de saúde sobre a prevenção da mortalidade materna por Hemorragia Pós-Parto (HPP), uma vez que objetiva-se contribuir para o diagnóstico e manejo precoce da HPP.

Outrossim, estudos futuros com essa tecnologia podem ser realizados para avaliar a retenção do conhecimento adquirido sobre prevenção da morte materna por Hemorragia Pós-Parto pelos estudantes de medicina, por meio de um questionário préteste para quantificar o conhecimento prévio sobre este tema, a aplicação da série de podcasts e um formulário pós-teste, para 
comparar as respostas antes e depois de ouvir os podcasts e poder analisar quais episódios conseguiram transmitir melhor este conhecimento, mas também em quais ajustes são necessários. E, por fim, enfatiza-se a importância da parceria com a Universidade do Estado do Pará (UEPA), campus VIII-Marabá para a reprodução e distribuição deste material educacional na versão podcast.

\section{Referências}

Coradini, N. H. K., Borges, A. F. \& Dutra, C. E. M. (2020). Tecnologia educacional podcast na educação profissional e Tecnológica. Revista Eletrônica Científica Ensino Interdisciplinar, 16 (6), 3-13.

Datasus, Departamento de informática do sus, Painel de Monitoramento da Mortalidade Materna (2019). Obtido em: http://svs.aids.gov.br/dantps/centrais-deconteudos/paineis demonitoramento/mortalidade/materna/.

Foschini, A. \& Taddei, R. (2006). Conquiste a rede - Podcast. Ebook.

Gomes, R. M. C. M., Alencar, M. P., Santos, M. J. M. N., da Silva, R. S., Messias, J. B., e Florêncio, M. S. (2019). Café com Saúde- Podcast como Ferramenta de Ensino nos Cursos de Saúde. In IV Congresso sobre Tecnologias na Educação (pp.1-9).

Leite, S. S. L., Áfio, A. C. E., Carvalho, L. V., Silva, J. M., Almeida, P. C. \& Pagliuca, L. M. F. (2018). Construção e validação de Instrumento de Validação de Conteúdo Educativo em Saúde. Rev Bras Enferm,4 (71), 1732-1738.

Lima, C. J. M., Coelho, R., Medeiros, M. S., Lubrusly, M., Marçal, E. \& Júnior, A. A. P. (2019). Desenvolvimento e Validação de um Aplicativo Móvel para o Ensino de Eletrocardiograma. Revista Brasileira de Educação Médica, 1 (43), 166-174.

Luiz, L. (2014). Reflexões sobre podcast. Marsupial.

Moudi, Z., Tabatabaei, S. M., Moakhar, A. S. \& Mollashahi, S. (2019). Empowering midwives to manage postpartum haemorrhage in rural areas of Islamic Republic of Iran: lessons learnt from cases of maternal death. EMHJ, 9 (25), 2-7.

Muniz, R. A. A. (2017). Construção e Validação de podcast com conteúdo educacional em saúde com participação ativa de acadêmicos de enfermagem. Dissertação de mestrado, Universidade Federal de Pernambuco, Recife, PE, Brasil.

OPAS-Organização Pan-Americana da Saúde (2018). Manual de orientação para o curso de prevenção de manejo obstétrico da hemorragia: Zero Morte Materna por Hemorragia. Brasilia: OPAS.

OPAS-Organização Pan-Americana da Saúde (2018). Recomendações assistenciais para prevenção, diagnóstico e tratamento da hemorragia obstétrica. Brasília: OPAS.

Perdigão, M. M. M., Rodrigues, A. B., Magalhães, T. L., Freitas, F. M. C., Bravo, L. G. \& Oliveira, P. P. (2019). Tecnologia educativa para manejo da fadiga relacionada à quimioterapia antineoplásica. Rev Bras Enferm, 72 (6), 1596-1602.

Pereira, A. S., Shitsuka, D. M., Parreira, F. J \& Shitsuka, R. (2018). Metodologia da pesquisa científica.

Rangel, R. C. T., Souza, M. L., Bentes, C. M. L., Souza, A. C. R. H., Leitão, M. N. C. \& Lynn, F. A. (2019). Care technologies to prevent and control hemorrhage in the third stage of labor: a systematic review. Rev. Latino-Am. Enfermagem, 27 (1), 2-10.

Saidelles, T., Minuzi, N. A., Barin, C. S. \& Santos, L. M. A. (2018). A utilização do podcast como uma ferramenta inovadora no contexto educacional. In $23^{\circ}$ seminário internacional de educação, tecnologia e sociedade (pp. 1-10).

Santos, F. E. P. (2020). Informação científica por meio da produção de conteúdo em podcast: hospedagem, distribuição e agregadores. ConCi- Conv. Ciênc. Inform., 2 (3), 22-51.

Santos, R. K., Moura, S. S. T., Souza, V. K. S. \& Sanches, L. M. P. (2016). Projeto "Conteiner Saúde-O uso do podcast como ferramenta educacional e populações vulneráveis. Um relato de experiência. In PROExC UFPE- 70 anos Tempos Transversos (pp. 1-3). Recife.

Saraiva, J. R., Rovadoschi, G., Menezes, A., Barreto, P. M., Konrath, T., Arbo, M. \& Franzoni, F. (2018). LasseCast-Produção de podcast como recurso pedagógico para cursos à distância. In Experiência inovadora/Materiais Didáticos para Universidade LaSalle EAD (pp. 1-10). Canoas.

Silva, M. S. (2019). O uso do podcast como recurso de aprendizagem em ensino superior. Dissertação de Mestrado, Universidade do Vale do Taquari., Lajeado, RS, Brasil.

Silva, S. S. F., Monteiro, J. A. S., Silva, L. R., Francisco, M. R., Brasil, Y. Z. \& Paula, D. G. (2020). Produções tecnológicas desenvolvidas por Enfermagem: revisão sistemática. Research, Society and Development, 8 (9), 2-33.

Vanassi, G. C. (2007). Podcasting como processo midiático interativo. Obtido em http://bit.ly/1n8lkgH.

Vasconcelos, V. M. S., Freitas, B. J. S., Martins, M. C. C., Oliveira, A. D. S., Viana, M. R. P., Mendes, C. M. M. \& Ramos, C. V. (2020). Validação de tecnologia educacional em saúde sobre "terapia nutricional enteral domiciliar" para cuidadores de idosos. Research, Society and Development, 10 (9), 8-16.

Veloso, C., Balduino I., Santos J., Marques J., Júnior, R., \& Rosa, R. (2019). Projeto Metacast: o uso do podcast como ferramenta de ensino-aprendizagem. In XX Congresso de Ciências da Comunicação na Região Sul (pp. 1-12). 